\section{Recovery from amnesia following transcorneal electroconvulsive shock*}

\author{
RALPH R. MILLER and ALAN D. SPRINGER \\ Brooklyn College of the City University of New York, Brooklyn, N.Y. 11210
}

Rats that received one-trial passive avoidance training, followed 8 sec later by transcorneal electroconvulsive shock (ECS), displayed extensive memory deficits on a 24-h retention test. However, a noncontingent footshock delivered outside of the training situation during the retention interval caused a partial recovery of memory. Control Ss receiving similar stimulation entirely outside of the training situation did not display equivalent avoidance behavior, suggesting that the avoidance behavior of the former group was specific to the training situation rather than a systemic effect of the combined treatments. These data indicate that transcorneal ECS is similar to transpinnate ECS in that both interfere with retrieval processes that normally occur soon after acquisition.

The permanence of amnesia induced by electroconvulsive shock (ECS) has been the subject of considerable research in recent years. Initial attention focused upon spontaneous recovery over prolonged retention intervals. However, while occasional spontaneous recovery studies yielded positive results (e.g., Zinkin \& Miller, $1967)$, the majority have found spontaneous recovery from ECS-induced amnesia to be a slight or nonexistent phenomenon (e.g., Luttges \& McGaugh, 1967; Herz \& Peeke, 1968). More recently, facilitated recovery of memory has been demonstrated in numerous instances (e.g., Koppenaal, Jagoda, \& Cruce, 1967; Quartermain, McEwen, \& Azmitia, 1970; Miller \& Springer, 1972). For example, Miller \& Springer (1972) found that noncontingent footshock (FS) delivered outside the training apparatus during the retention period could induce substantial recovery of memory. In this instance, recovery was independent of the interval between ECS and noncontingent FS. The recovery phenomenon indicates that memories ordinarily not evident after ECS may be present but unavailable. This view is in contrast with the traditional view that ECS interferes with consolidation processes, thereby causing irreversible amnesia.

Assuming that some amnesia is clearly reversible, parsimony in the form of a uniprocess model would suggest that all amnesia is similarly reversible, at least until contrary evidence is presented. It is not clear whether the usual lack of complete recovery is due to the use of less than ideal parameters for facilitating recovery or to an irreversible consequence of ECS. King \& Glasser

* Supported by USPHS Research Grant MH 19497 and Research Foundation of CUNY Research Grant 1385.
(1970) argue for a dual-process model of ECS-induced amnesia in which permanent amnesia is produced when ECS "is of sufficient intensity and is effectively delivered so as to produce a complete disruption of consolidation processes." Unfortunately, their only definition of sufficient intensity and effective delivery is that permanent amnesia be produced. Noting that transcorneal ECS ordinarily produces a more profound amnesia than transpinnate ECS, King and Glasser suggest that transcorneal electrodes constitute a more effective method of delivering ECS than do transpinnate electrodes. (It is not clear whether the greater effectiveness of transcorneal ECS is due to the neuroanatomical pathway of the convulsive current and/or to a greater percentage of the applied current actually reaching neural tissue.) The present experiment investigates the reversibility of amnesia induced by moderately high-intensity transcorneal ECS.

\section{SUBJECTS AND APPARATUS}

Seventy naive male albino rats of the Sprague-Dawley strain were obtained from Sandra Biological Laboratories (New York, N.Y.). All animals were 60-70 days old and weighed $170-210 \mathrm{~g}$ on the day of training. Ss were individually housed in continuous light and maintained on ad lib water and $10 \mathrm{~g} / \mathrm{day}$ of Purina Rat Chow, which, on experimental days, was given $1 \mathrm{~h}$ after treatment. Ss were handled for $1 \mathrm{~min} /$ day several times a week during preexperimental adaptation to the colony environment. Each $\mathbf{S}$ was assigned randomly to one of three groups.

The apparatus was modeled after the Jarvik \& Kopp (1967) step-through device, but modified to accommodate rats rather than mice. The floor of the device was a linear runway, $5 \mathrm{~cm}$ wide, composed of two parallel metal plates, each $2 \mathrm{~cm}$ wide, with a 1-cm gap between them. The metal side walls slanted outward as they extended up from the floor, so that the compartment was $20 \mathrm{~cm}$ wide at the $28-\mathrm{cm}$-high Plexiglas ceiling. The device was $60 \mathrm{~cm}$ long and had Plexiglas vertical end walls and an opaque gated partition which divided the step-through apparatus into a 16-cm-long white start chamber and a 44-cm-long black punishment chamber. A $4.5-\mathrm{cm}$ hurdle at the gate was used to add distinctiveness to the step-through response. The only illumination in the apparatus was a $25-W$ incandescent bulb positioned behind the end of the start compartment, $20 \mathrm{~cm}$ above the compartment floor. This served as a mildly aversive stimulus which insured a rather low operant step-through latency. The two floor plates and contiguous side walls of the black box were connected to a Lafayette Model 82405 constant-current shock source calibrated to deliver a 2.0-rms-mA $60-\mathrm{Hz}$ FS.

A $50 \times 50 \mathrm{~cm}$ open field was used as the location for FS and ECS in the absence of step-through apparatus cues. To minimize the similarity between the step-through apparatus and the open field, the floor of the open field was a grid of stainless steel rods, $0.55 \mathrm{~cm}$ in diam. Preliminary observations indicated that an $0.7-\mathrm{rms}-\mathrm{mA}$ FS in the open field produced as much jumping and vocalizing as a $2.0-\mathrm{mA}$ FS in the step-through device. This was probably due to the greater sensitivity of the instep of the paw than that of the tip of the toe, the former being the point of contact on a grid floor and the latter being the point of contact on the metal plate floor of the step-through device. Thus, open-field FS was $0.7 \mathrm{~mA}$ in this experiment. Control groups demonstrating the appropriateness of equating $2.0 \mathrm{~mA}$ in the step-through apparatus with $0.7 \mathrm{~mA}$ in the open field may be found elsewhere (Miller \& Springer, 1972).

The FS serving as a potential recovery agent was administered in a Grason-Stadler Model 1110L operant chamber (less bar) with the houselight connected and the fan disconnected. The $30 \times 30 \mathrm{~cm}$ grid floor was wired to deliver a $0.7-\mathrm{mA}$ FS. This intensity was determined by the same method used in the open field.

ECS was provided by a Lafayette Model 82403 constant-current shock source calibrated to deliver $50 \mathrm{rms} \mathrm{mA}$ at $60 \mathrm{~Hz}$. The observed range of actual intensities as monitored on an oscilloscope was $45.55 \mathrm{~mA}$. PROCEDURE

Group E (N = 24), the basic experimental group, was placed in the white compartment of the step-through device facing away from 
the gate. Three seconds later, the gate was opened and a clock was started. Upon entering the black compartment (criterion of a response was four paws in the black compartment), the gate was closed, the response latency was recorded, and the $\mathrm{S}$ was given $10 \mathrm{sec}$ of FS. At FS offset, each animal was immediately lifted and given $200 \mathrm{msec}$ of transcorneal ECS in the E's hand. The mean FS-ECS interval was $8 \mathrm{sec}$. Two hours later, each animal was placed in the operant chamber and received a 10 -sec FS (noncontingent in respect to the step-through device). Twenty-two hours later, step-through latencies in the training apparatus were obtained by a procedure identical to training, except that neither FS nor ECS was administered. A 300-sec ceiling was in effect on the test trials. The 2-h interval between training and operant-chamber-FS was in considerable excess of the post-ECS interval in which memory has been observed to linger in rats prior to the development of amnesia (Miller \& Springer, 1971).

Group A $(N=23)$, the amnestic control group, was treated identically to Group E, except that pseudo-FS was administered in the operant chamber.

On the training day, Group $S(N=$ $23)$, the systemic control group, received 10 sec of FS in the open field, followed approximately $8 \mathrm{sec}$ later by $200 \mathrm{msec}$ of transcorneal ECS. Two hours after this treatment, these Ss received $10-\mathrm{sec}$ of noncontingent shock in the operant chamber, which in turn was followed in $22 \mathrm{~h}$ by a test trial in the step-through apparatus.

All latencies were transformed to $\log$ latencies to permit the use of parametric statistical analysis.

$A$ group receiving training $F S$ without ECS was not included in this experiment as numerous other studies (e.g., Miller \& Springer, 1971, 1972) have found that such groups invariably produce mean step-through latencies of $65-100 \mathrm{sec}(\overline{\mathrm{X}}=1.80$ to $2.00 \mathrm{log}$ $\mathrm{sec}$ ).

\section{RESULTS}

Group mean training trial scores were: Group $\mathrm{E}=0.95 \mathrm{log} \mathrm{sec}(8.9 \mathrm{sec})$, Group $\mathrm{A}=1.05 \log \mathrm{sec}(11.2 \mathrm{sec})$, and Group $S=1.05 \mathrm{log} \mathrm{sec}(11.2 \mathrm{sec})$. Consistent with there being no group differences prior to treatment, analysis of these means proved nonsignificant $(\mathrm{F}=0.55, \mathrm{df}=2,67, \mathrm{p}>.25)$. Group mean test trial scores were: Group $\mathrm{E}=$ $1.35 \mathrm{log} \mathrm{sec}(22.4 \mathrm{sec})$, Group $\mathrm{A}=$ $1.02 \log \mathrm{sec}(10.5 \mathrm{sec})$, and Group $\mathrm{S}=$ $0.96 \mathrm{log} \mathrm{sec}(9.1 \mathrm{sec})$. Analysis of variance found these means to differ significantly $(\mathrm{F}=3.42, \mathrm{df}=2,67$, $\mathrm{p}<.05$ ). Individual two-tailed $t$ tests, using the within-cell variance from the test trial ANOVA to estimate the standard error, found significant differences between Groups $E$ and $A$ ( $t$ $=2.89$, df $=45, p<.01$, Groups $E$ and $S(t=3.43, \mathrm{df}=45, \mathrm{p}<.01)$, but not Groups $A$ and $S(t=0.53, d f=44$, $\mathrm{p}>.50$ ).

The similarity of Group A training and test trial scores indicates an essentially complete amnesia for training FS. However, a noncontingent FS between training and retention appears to have induced significant recovery of memory in Group $\mathrm{E}$. As Group E scores are appreciably lower than those of trained animals that had never received ECS in other studies $(\bar{X}$ $=1.80$ to $2.00 \log$ sec; see Miller \& Springer, 1971, 1972), recovery was obviously only partial. The low test scores of Group S, which received FS + ECS plus FS during the retention interval, with intensities, durations, and sequence equivalent to those of Group E, indicate that the relatively high Group E test scores are specific to the training situation rather than due to a systemic effect independent of situational cues.

\section{DISCUSSION}

The present data demonstrate that amnesia induced by transcorneal ECS can be reversed by a noncontingent FS administered during the retention interval. However, as previously noted, recovery of memory was not complete. Either the optimal parameters for facilitated recovery were not used or amnesia is due to both a reversible factor and a non reversible factor. The former position suggests a uniprocess model, while the latter position minimally requires a dual process model. In the interest of parsimony, the authors are presently inclined to favor a uniprocess hypothesis. It appears unlikely that any retroactive amnesia paradigm presently in use will ever provide support for a dual process model, for in any given instance, amnesia will either prove reversible or be "not as yet reversed." The experimental design relegates irreversibility to the status of a null hypothesis.

The traditional consolidation explanation of ECS-induced amnesia proposes that ECS administered soon after acquisition prevents the formation of long-term storage (McGaugh, 1966). If this hypothesis were correct, amnesia observed long after training should be permanent. The present data indicate that amnesia is not necessarily permanent, thus leading the authors to believe that ECS-induced amnesia represents a retrieval failure rather than a storage (consolidation) failure. Given the well-known retrograde gradient of ECS-induced amnesia, the proposed retrieval failure position must assume that there are not only storage processes but also retrieval processes occurring at the time of and/or soon after acquisition. Such retrieval processes might be analogous to cataloging, which facilitates access to the relevant information during subsequent test trials, and are assumed distinguishable from consolidation processes which are defined here to concerm only the formation of the underlying structural-chemical engram of long-term storage. This view of retrieval failure has been used by numerous other researchers (e.g., Koppenaal, Jagoda, \& Cruce, 1967; Quartermain, McEwen, \& Azmitia, 1970) and should not be confused with the categorical definitions of storage being all processes occurring during and soon after acquisition, and retrieval being all processes occurring at the time of testing (e.g., Cherkin, 1970). The latter set of definitions requires that ECS-induced amnesia be classified as a storage (consolidation) failure due to the nature of the retrograde gradient of amnesia.

The conditions under which induced recovery from amnesia will occur are far from clear. Initial attempts to explain the phenomenon spoke of "reminder" events (e.g., Lewis, Misanin, \& Miller, 1968) assuming a necessary stimulus similarity of the recovery agent to the initial training situation. More recently, Springer \& Miller (1972) have successfully used noncontingent FS to induce recovery of memory of ice-water immersion. Their data indicate that the recovery agent need not be stimulus specific with respect to acquisition cues.

REFERENCES
CHERKIN, A. Retrograde amnesia Impaired memory consolidation or impaired retrieval? Communications in Behavioral Biology, 1970, A, 5, 183-190.

HERZ M. J., \& PEEKE, H. V. S. ECS-produced retrograde amnesia: Permanence vs. recovery over repeated testing. Physiology \& Behavior, 1968, 3. $517-521$.

JARVIK, M. E., \& KOPP, R. An improved one-trial passive avoidance learning situation. Psychological Reports, 1967, 21, 221-224.

KING, R. A., \& GLASSER, R. L. Duration of electroconvulsive shock-induced retrograde amnesia in rats. Physiology \& Behavior, $1970,5,335-340$.

KOPPENAAL, R. J., JAGODA, E., \& CRUCE, J. A. $\vec{F}$, Recovery from ECS-produced amnesia following a reminder. Psychonomic Science, 1967, 9, 293-294.

LEWIS, D. J., MISANIN, J. R., \& MILLER, R. R. Recovery of memory following amnesia. Nature, $1968,220,704-705$.

LUTTGES, M. W., \& McGAUGH, J. L. Permanence of retrograde amnesia produced by electroconvulsive shock. Science, 1967, 156, 408-410.

McGAUGH, J. L. Time dependent processes in memory storage. Science, 1966, 153, 1351-1358. 
MILLER, R. R., \& SPRINGER, A. D. Temporal course of amnesia in rats after electroconvulsive shock. Physiology \& Behavior, 1971, 6, 229-233.

MILLER, R. R., \& SPRINGER, A. D. Induced recovery of memory in rats following ECS. Physiology \& Behavior,
1972 , in press.

QUARTERMAIN, D., MCEWEN, B. S., \& AZMITIA, E. C.. JR. Amnesia produced by electroconvulsive shock or cycloheximide: Conditions for recovery. Science, $1970,169,683-686$

SPRINGER, A. D., \& MILLER, $R, R$.
Recovery of memory in the rat following ECS using dissimilar training and recovery agents. Science, 1972 , in press.

ZINKIN, S., \& MILLER, A. J. Recovery of memory after amnesia induced by electroconvulsive shock. Science, 1967. 155, 102-104. 